\title{
Persistence properties of solutions to the dissipative 2-component Degasperis-Procesi system
}

Sen Ming ${ }^{1}$, Han Yang ${ }^{1 *}$ and Ls Yong ${ }^{2}$

"Correspondence:

hanyang95@263.net

'School of Mathematics, Southwest

Jiaotong University, Chengdu,

610031, China

Full list of author information is

available at the end of the article

\begin{abstract}
The persistence properties of solutions to the dissipative 2-component Degasperis-Procesi system are investigated. We find that if the initial data with their derivatives of the system exponentially decay at infinity, then the corresponding solution also exponentially decays at infinity.

MSC: $35 \mathrm{G} 25 ; 35 \mathrm{~L} 15 ; 35 \mathrm{Q} 58$
\end{abstract}

Keywords: 2-component; Degasperis-Procesi system; dissipative; persistence properties

\section{Introduction}

We consider the following dissipative 2-component Degasperis-Procesi system:

$$
\begin{cases}u_{t}-u_{x x t}+4 u u_{x}+\lambda_{1}\left(u-u_{x x}\right)+c \rho \rho_{x}=3 u_{x} u_{x x}+u u_{x x x}, & t>0, x \in \mathbb{R}, \\ \rho_{t}+u \rho_{x}+2 u_{x} \rho+\lambda \rho=0, & t>0, x \in \mathbb{R}, \\ u(0, x)=u_{0}(x), \quad \rho(0, x)=\rho_{0}(x), & x \in \mathbb{R},\end{cases}
$$

where $\lambda, \lambda_{1}$ are nonnegative constants, $c \in \mathbb{R},\left(u_{0}, \rho_{0}\right) \in H^{s}(\mathbb{R}) \times H^{s-1}(\mathbb{R})\left(s>\frac{3}{2}\right)$.

In system (1.1), if $\lambda_{1}=\rho=0$, we get the classical Degasperis-Procesi equation [1]

$$
u_{t}-u_{x x t}+4 u u_{x}=3 u_{x} u_{x x}+u u_{x x x}
$$

where $u(t, x)$ represents the fluid velocity at time $t$ in $x$ direction and $k \in \mathbb{R}$. The nonlinear convection term $u u_{x}$ causes the steepening of wave form. The nonlinear dispersion effect term $3 u_{x} u_{x x}+u u_{x x x}$ makes the wave form spread. The Degasperis-Procesi equation has been studied in many works [2-8]. Escher et al. [2] demonstrated that there exists a unique solution $u(t, x) \in C\left([0, T], H^{s}(\mathbb{R})\right) \cap C^{1}\left([0, T], H^{s-1}(\mathbb{R})\right)$ to $(1.2)$ with initial value $u_{0}(x) \in H^{s}(\mathbb{R})\left(s>\frac{3}{2}\right)$. Liu and Yin [3] obtained the global existence of solutions to (1.2). They derived several wave breaking mechanisms in Sobolev space $H^{s}(\mathbb{R})$ with $s>\frac{3}{2}$. Yin [4] established the local well-posedness for the Degasperis-Procesi equation with initial value $u_{0} \in H^{s}(\mathbb{R})\left(s>\frac{3}{2}\right)$ on the line. In [5], the author obtained the global existence of solutions to the Degasperis-Procesi equation on the circle. The precise blow-up scenario was also derived. The global existence of strong solutions and global weak solutions to the

\section{焦 Springer}

@2014 Ming et al.; licensee Springer. This is an Open Access article distributed under the terms of the Creative Commons Attribution License (http://creativecommons.org/licenses/by/2.0), which permits unrestricted use, distribution, and reproduction in any medium, provided the original work is properly cited. 
Degasperis-Procesi equation were shown in [6, 7]. Guo et al. [9] studied the dissipative Degasperis-Procesi equation,

$$
u_{t}-u_{x x t}+4 u u_{x}+\lambda\left(u-u_{x x}\right)=3 u_{x} u_{x x}+u u_{x x x}
$$

where $\lambda\left(u-u_{x x}\right)(\lambda>0)$ is the dissipative term. They obtained the global weak solutions to (1.3). Guo [10] established the local well-posedness for (1.3), and also obtained the global existence, persistence properties and propagation speed of solutions. Wu and Yin [8] obtained the local well-posedness for (1.3), and also studied the blow-up scenarios of solutions in periodic case.

On the other hand, many researchers have studied the integrable multi-component generalizations of the Degasperis-Procesi equation [11-16]. Yan and Yin [11] investigated the 2-component Degasperis-Procesi system

$$
\begin{cases}u_{t}-u_{x x t}+4 u u_{x}+c \rho \rho_{x}=3 u_{x} u_{x x}+u u_{x x x}, & t>0, x \in \mathbb{R} \\ \rho_{t}+u \rho_{x}+2 u_{x} \rho=0, & t>0, x \in \mathbb{R} \\ u(0, x)=u_{0}(x), \quad \rho(0, x)=\rho_{0}(x), & x \in \mathbb{R},\end{cases}
$$

where $c \in \mathbb{R}$. They established the local well-posedness for system (1.4) in Besov space $B_{p, r}^{s}(\mathbb{R}) \times B_{p, r}^{s-1}(\mathbb{R})$ with $s>\max \left(1+\frac{1}{p}, \frac{3}{2}\right)$, and also derived the precise blow-up scenarios of strong solutions in Sobolev space $H^{s}(\mathbb{R}) \times H^{s-1}(\mathbb{R})$ with $s>\frac{3}{2}$. Zhou et al. [12] investigated the traveling wave solutions to the 2-component Degasperis-Procesi system. Manwai [16] studied the self-similar solutions to the 2-component Degasperis-Procesi system. Fu and $\mathrm{Qu}$ [13] obtained the persistence properties of solutions to the 2-component DegasperisProcesi system in Sobolev space $H^{s}(\mathbb{R}) \times H^{s-1}(\mathbb{R})$ with $s \geq 2$. For system (1.4), Jin and Guo [14] studied the blow-up mechanisms and persistence properties of strong solutions.

Recently, a large amount of literature has been devoted to the study of the 2-component Camassa-Holm system [17-28]. Hu [18] studied the dissipative periodic 2-component Camassa-Holm system

$$
\begin{cases}u_{t}-u_{x x t}+3 u u_{x}+\lambda\left(u-u_{x x}\right)+\rho \rho_{x}=2 u_{x} u_{x x}+u u_{x x x}, & t>0, x \in \mathbb{S} \\ \rho_{t}+u \rho_{x}+u_{x} \rho+\lambda \rho=0, & t>0, x \in \mathbb{S} \\ u(t, x+1)=u(t, x), \quad \rho(t, x+1)=\rho(t, x), & t>0, x \in \mathbb{S} \\ u(0, x)=u_{0}(x), \quad \rho(0, x)=\rho_{0}(x), & x \in \mathbb{S},\end{cases}
$$

where $\lambda>0$. The author not only established the local well-posedness for system (1.5) in Besov space $B_{p, r}^{s}(\mathbb{S}) \times B_{p, r}^{s-1}(\mathbb{S})$ with $s>\max \left(1+\frac{1}{p}, \frac{3}{2}\right)$, but she also presented global existence results and the exact blow-up scenarios of strong solutions in Sobolev space $H^{s}(\mathbb{S}) \times H^{s-1}(\mathbb{S})$ with $s>\frac{3}{2}$. For $\lambda=0$ in system (1.5), Jin and Guo [19] considered the persistence properties of solutions to the modified 2-component Camassa-Holm system. Zhu [20] considered the persistence property of solutions to the coupled Camassa-Holm system, and also established the global existence and blow-up mechanisms of solutions. Guo $[21,22]$ studied the persistence properties and unique continuation of solutions to the 2 -component Camassa-Holm system in the case $\lambda=0$. It was shown in [29] that the dissipative Camassa-Holm, Degasperis-Procesi, Hunter-Saxton and Novikov equations could be reduced to the non-dissipative versions by means of an exponentially time-dependent 
scaling. One may refer to [30-34] and the references therein for more details in this direction.

Motivated by the work in [13, 20,35], we study the dissipative 2-component DegasperisProcesi system (1.1). We note that the persistence properties of solutions to system (1.1) have not been discussed yet. The aim of this paper is to investigate the persistence properties of solutions in Sobolev space $L^{\infty}(\mathbb{R})$. The main idea of this work comes from [35].

Now we rewrite system (1.1) as

$$
\begin{cases}u_{t}+u u_{x}=P(D)\left(\frac{3}{2} u^{2}+\frac{1}{2} c \rho^{2}\right)-\lambda_{1} u, & t>0, x \in \mathbb{R}, \\ \rho_{t}+u \rho_{x}=-2 u_{x} \rho-\lambda \rho, & t>0, x \in \mathbb{R}, \\ u(0, x)=u_{0}(x), \quad \rho(0, x)=\rho_{0}(x), & x \in \mathbb{R},\end{cases}
$$

where the operator $P(D)=-\partial_{x}\left(1-\partial_{x}^{2}\right)^{-1}$.

The main results are presented as follows.

Theorem 1.1 Assume $T>0$ and $\left(u_{0}, \rho_{0}\right) \in H^{s}(\mathbb{R}) \times H^{s-1}(\mathbb{R})$ with $s>\frac{3}{2}$. Then the Cauchy problem $(1.1)$ has a unique solution $(u, \rho) \in C\left([0, T] ; H^{s}(\mathbb{R})\right) \cap C^{1}\left([0, T] ; H^{s-1}(\mathbb{R})\right) \times$ $C\left([0, T] ; H^{s-1}(\mathbb{R})\right) \cap C^{1}\left([0, T] ; H^{s-2}(\mathbb{R})\right)$.

Theorem 1.2 Let $T>0$ and $\left(u_{0}, \rho_{0}\right) \in H^{s}(\mathbb{R}) \times H^{s-1}(\mathbb{R})$ with $s>\frac{3}{2} .(u, \rho) \in C([0, T]$; $\left.H^{s}(\mathbb{R})\right) \cap C^{1}\left([0, T] ; H^{s-1}(\mathbb{R})\right) \times C\left([0, T] ; H^{s-1}(\mathbb{R})\right) \cap C^{1}\left([0, T] ; H^{s-2}(\mathbb{R})\right)$ is the corresponding solution to system (1.1). If there exists $\theta \in(0,1)$ such that

$$
\left|u_{0}(x)\right|,\left|\partial_{x} u_{0}(x)\right|,\left|\rho_{0}(x)\right| \sim O\left(e^{-\theta x}\right) \quad \text { as } x \rightarrow \infty,
$$

then

$$
|u(t, x)|,\left|\partial_{x} u(t, x)\right|,|\rho(t, x)| \sim O\left(e^{-\theta x}\right) \quad \text { as } x \rightarrow \infty
$$

uniformly on the interval $[0, T]$.

Theorem 1.3 Let $T>0$ and $\left(u_{0}, \rho_{0}\right) \in H^{s}(\mathbb{R}) \times H^{s-1}(\mathbb{R})$ with $s>\frac{3}{2} .(u, \rho) \in C([0, T]$; $\left.H^{s}(\mathbb{R})\right) \cap C^{1}\left([0, T] ; H^{s-1}(\mathbb{R})\right) \times C\left([0, T] ; H^{s-1}(\mathbb{R})\right) \cap C^{1}\left([0, T] ; H^{s-2}(\mathbb{R})\right)$ is the corresponding solution to system (1.1). Assume the constant $\theta \in\left(\frac{1}{2}, 1\right)$.

(1) For $c>0$,

$$
\left|u_{0}(x)\right| \sim o\left(e^{-x}\right), \quad\left|\partial_{x} u_{0}(x)\right| \sim O\left(e^{-\theta x}\right), \quad\left|\rho_{0}(x)\right| \sim O\left(e^{-\theta x}\right) \quad \text { as } x \rightarrow \infty,
$$

and there exists $t_{1} \in(0, T)$ such that $\left|u\left(t_{1}, x\right)\right| \sim o\left(e^{-x}\right)$ as $x \rightarrow \infty$, then

$$
u(t, x)=0, \quad \rho(t, x)=0 .
$$

(2) For $c=0$,

$$
\left|u_{0}(x)\right| \sim o\left(e^{-x}\right), \quad\left|\partial_{x} u_{0}(x)\right| \sim O\left(e^{-\theta x}\right) \quad \text { as } x \rightarrow \infty,
$$

and there exists $t_{1} \in(0, T)$ such that $\left|u\left(t_{1}, x\right)\right| \sim o\left(e^{-x}\right)$ as $x \rightarrow \infty$, then

$$
u(t, x)=0, \quad \rho(t, x)=\rho_{0}(x) e^{-\lambda t} .
$$


Theorem 1.4 Let $\lambda_{1}=0$ in system (1.1). Assume $T>0$ and $\left(u_{0}, \rho_{0}\right) \in H^{s}(\mathbb{R}) \times H^{s-1}(\mathbb{R})$ with $s>\frac{3}{2}$. $(u, \rho) \in C\left([0, T] ; H^{s}(\mathbb{R})\right) \cap C^{1}\left([0, T] ; H^{s-1}(\mathbb{R})\right) \times C\left([0, T] ; H^{s-1}(\mathbb{R})\right) \cap C^{1}([0, T] ;$ $\left.H^{s-2}(\mathbb{R})\right)$ is the corresponding solution to system (1.1). If there exists $\theta \in\left(\frac{1}{2}, 1\right)$ such that

$$
\left|u_{0}(x)\right| \sim O\left(e^{-x}\right), \quad\left|\partial_{x} u_{0}(x)\right| \sim O\left(e^{-\theta x}\right), \quad\left|\rho_{0}(x)\right| \sim O\left(e^{-\theta x}\right) \quad \text { as } x \rightarrow \infty,
$$

then

$$
|u(t, x)| \sim O\left(e^{-x}\right), \quad|\rho(t, x)| \sim O\left(e^{-\theta x}\right) \quad \text { as } x \rightarrow \infty
$$

uniformly on the interval $[0, T]$.

Theorem 1.5 Assume $T>0$ and $\left(u_{0}, \rho_{0}\right) \in H^{s}(\mathbb{R}) \times H^{s-1}(\mathbb{R})$ with $s>\frac{5}{2} .(u, \rho) \in C([0, T]$; $\left.H^{s}(\mathbb{R})\right) \cap C^{1}\left([0, T] ; H^{s-1}(\mathbb{R})\right) \times C\left([0, T] ; H^{s-1}(\mathbb{R})\right) \cap C^{1}\left([0, T] ; H^{s-2}(\mathbb{R})\right)$ is the corresponding solution to system (1.1). If there exists $\theta \in(0,1)$ such that

$$
\left|u_{0}(x)\right|,\left|\rho_{0}(x)\right|,\left|u_{0 x}(x)\right|,\left|\rho_{0 x}(x)\right| \sim O\left(e^{-\theta x}\right) \quad \text { as } x \rightarrow \infty,
$$

then

$$
|u(t, x)|,|\rho(t, x)|,\left|u_{x}(t, x)\right|,\left|\rho_{x}(t, x)\right| \sim O\left(e^{-\theta x}\right) \quad \text { as } x \rightarrow \infty .
$$

Theorem 1.6 Assume $T>0$ and $\left(u_{0}, \rho_{0}\right) \in H^{s}(\mathbb{R}) \times H^{s-1}(\mathbb{R})$ with $s>\frac{5}{2}$. $(u, \rho) \in C([0, T]$; $\left.H^{s}(\mathbb{R})\right) \cap C^{1}\left([0, T] ; H^{s-1}(\mathbb{R})\right) \times C\left([0, T] ; H^{s-1}(\mathbb{R})\right) \cap C^{1}\left([0, T] ; H^{s-2}(\mathbb{R})\right)$ is the corresponding solution to system (1.1). If there exists $\theta \in\left(\frac{1}{2}, 1\right)$ such that

$$
\left|u_{0}(x)\right|,\left|\rho_{0}(x)\right| \sim o\left(e^{-x}\right) \quad \text { as } x \rightarrow \infty, \quad\left|u_{0 x}(x)\right|,\left|\rho_{0 x}(x)\right| \sim O\left(e^{-\theta x}\right) \quad \text { as } x \rightarrow \infty,
$$

and there exists $t_{1} \in[0, T]$ such that

$$
\left|u\left(t_{1}, x\right)\right|,\left|\rho\left(t_{1}, x\right)\right| \sim o\left(e^{-x}\right) \quad \text { as } x \rightarrow \infty,
$$

then

$$
u(t, x)=0, \quad \rho(t, x)=0 .
$$

The remainder of this paper is organized as follows. In Section 2, the proofs of Theorems 1.1 and 1.2 are presented. Section 3 is devoted to the proofs of Theorems 1.3 and 1.4. The proofs of Theorems 1.5 and 1.6 are given in Section 4.

Notation We denote the norm of Lebesgue space $L^{p}(\mathbb{R}), 1 \leq p \leq \infty$ by $\|\cdot\|_{L^{p}}$, the norm in Sobolev space $H^{s}(\mathbb{R}), s \in \mathbb{R}$ by $\|\cdot\|_{H^{s}}$ and the norm in Besov space $B_{p, r}^{s}(\mathbb{R}), s \in \mathbb{R}$ by $\|\cdot\|_{B_{p, r}^{s}}$. For $\theta \in \mathbb{R}$, we denote

$$
\begin{aligned}
& |f(x)| \sim O\left(e^{\theta x}\right) \quad \text { as } x \rightarrow \infty, \text { if } \lim _{x \rightarrow \infty} \frac{|f(x)|}{e^{\theta x}}=L(L \neq 0) ; \\
& |f(x)| \sim o\left(e^{\theta x}\right) \quad \text { as } x \rightarrow \infty, \text { if } \lim _{x \rightarrow \infty} \frac{|f(x)|}{e^{\theta x}}=0 .
\end{aligned}
$$




\section{Proofs of Theorems 1.1 and 1.2}

We write the definition of Besov space. One may check [36-39] for more details.

Proposition 2.1 [39] Let $s \in \mathbb{R}$ and $1 \leq p, r \leq \infty$. The nonhomogeneous Besov space is defined by $B_{p, r}^{s}(\mathbb{R})=\left\{f \in S^{\prime}(\mathbb{R}) \mid\|f\|_{B_{p, r}^{s}(\mathbb{R})}<\infty\right\}$, where

$$
\|f\|_{B_{, r}^{s}(\mathbb{R})}= \begin{cases}\left(\sum_{j=-1}^{\infty} 2^{j r s}\left\|\Delta_{j} f\right\|_{L^{p}}^{r}\right)^{\frac{1}{r}}, & r<\infty, \\ \sup _{j \geq-1} 2^{j s}\left\|\Delta_{j} f\right\|_{L^{p}}, & r=\infty .\end{cases}
$$

\subsection{Proof of Theorem 1.1}

Using the Littlewood-Paley theory and estimates for solutions to the transport equation, one may follow similar arguments as in [11] to establish the local well-posedness for system (1.1) with some modification. Here we omit the detailed proof. For system (1.1) with initial data $\left(u_{0}, \rho_{0}\right) \in B_{p, r}^{s} \times B_{p, r}^{s-1}\left(s>\max \left(1+\frac{1}{p}, \frac{3}{2}\right)\right)$, we see that the corresponding solution $(u, \rho) \in$ $C\left([0, T] ; B_{p, r}^{s}\right) \cap C^{1}\left([0, T] ; B_{p, r}^{s-1}\right) \times C\left([0, T] ; B_{p, r}^{s-1}\right) \cap C^{1}\left([0, T] ; B_{p, r}^{s-2}\right)$. Thus we complete the proof of Theorem 1.1.

\subsection{Proof of Theorem 1.2}

We denote

$$
F(u, \rho)=\frac{3}{2} u^{2}+\frac{c}{2} \rho^{2}, \quad M=\sup _{t \in[0, T]}\|(u, \rho)\|_{H^{s} \times H^{s-1}} .
$$

Multiplying the second equation in (1.6) by $\rho^{2 n-1}$ with $n \in \mathbb{N}^{*}$ and integrating the resultant equation with respect to $x$ yield

$$
\int_{\mathbb{R}} \rho_{t} \rho^{2 n-1} \mathrm{~d} x+\int_{\mathbb{R}} u \rho_{x} \rho^{2 n-1} \mathrm{~d} x+2 \int_{\mathbb{R}} u_{x} \rho \rho^{2 n-1} \mathrm{~d} x+\lambda \int_{\mathbb{R}} \rho \rho^{2 n-1} \mathrm{~d} x=0 .
$$

We have

$$
\begin{aligned}
& \int_{\mathbb{R}} \rho_{t} \rho^{2 n-1} \mathrm{~d} x=\frac{1}{2 n} \frac{\mathrm{d}}{\mathrm{d} t}\|\rho(t)\|_{L^{2 n}}^{2 n}=\|\rho(t)\|_{L^{2 n}}^{2 n-1} \frac{\mathrm{d}}{\mathrm{d} t}\|\rho(t)\|_{L^{2 n}}, \\
& \int_{\mathbb{R}} u \rho_{x} \rho^{2 n-1} \mathrm{~d} x+2 \int_{\mathbb{R}} u_{x} \rho \rho^{2 n-1} \mathrm{~d} x=-\frac{1}{2 n} \int_{\mathbb{R}} u_{x} \rho^{2 n} \mathrm{~d} x+2 \int_{\mathbb{R}} u_{x} \rho^{2 n} \mathrm{~d} x \\
& \leq\left(2-\frac{1}{2 n}\right)\left\|u_{x}(t)\right\|_{L^{\infty}}\|\rho(t)\|_{L^{2 n}}^{2 n}, \\
& \lambda \int_{\mathbb{R}} \rho \rho^{2 n-1} \mathrm{~d} x=\lambda\|\rho(t)\|_{L^{2 n}}^{2 n} .
\end{aligned}
$$

Thus

$$
\frac{\mathrm{d}}{\mathrm{d} t}\|\rho(t)\|_{L^{2 n}} \leq\left[\left(2-\frac{1}{2 n}\right)\left\|u_{x}(t)\right\|_{L^{\infty}}+\lambda\right]\|\rho(t)\|_{L^{2 n}} .
$$

If $s>\frac{3}{2}$, using the Sobolev embedding theorem, we have $\left\|u_{x}(t)\right\|_{L^{\infty}} \leq\|u(t)\|_{H^{s}} \leq M$. Applying the Gronwall inequality to (2.2) yields

$$
\|\rho(t)\|_{L^{2 n}} \leq\left\|\rho_{0}\right\|_{L^{2 n}} e^{\left[\left(2-\frac{1}{2 n}\right) M+\lambda\right] t} .
$$


Noting $u \in L^{2} \cap L^{\infty}$ gives rise to

$$
\lim _{p \rightarrow \infty}\|u\|_{L^{p}}=\|u\|_{L^{\infty}}
$$

and, taking the limit as $n \rightarrow \infty$, we obtain

$$
\|\rho(t)\|_{L^{\infty}} \leq\left\|\rho_{0}\right\|_{L^{\infty}} e^{(2 M+\lambda) t} .
$$

Multiplying the first equation in system (1.6) by $u^{2 n-1}$ with $n \in \mathbb{N}^{*}$ and integrating the resultant equation with respect to $x$ yield

$$
\int_{\mathbb{R}} u_{t} u^{2 n-1} \mathrm{~d} x+\int_{\mathbb{R}} u u_{x} u^{2 n-1} \mathrm{~d} x+\lambda_{1} \int_{\mathbb{R}} u u^{2 n-1} \mathrm{~d} x+\int_{\mathbb{R}} \partial_{x} g * F(u, \rho) u^{2 n-1} \mathrm{~d} x=0 .
$$

Using the Holder inequality, we have

$$
\int_{\mathbb{R}} \partial_{x} g * F(u, \rho) u^{2 n-1} \mathrm{~d} x \leq\left\|\partial_{x} g * F(u, \rho)\right\|_{L^{2 n}}\|u\|_{L^{2 n}}^{2 n-1},
$$

which in combination with (2.3) yields

$$
\frac{\mathrm{d}}{\mathrm{d} t}\|u\|_{L^{2 n}} \leq \lambda_{1}\|u\|_{L^{2 n}}+\left\|\partial_{x} g * F(u, \rho)\right\|_{L^{2 n}} .
$$

Using the Gronwall inequality, one derives

$$
\|u(t)\|_{L^{2 n}} \leq\left(\left\|u_{0}\right\|_{L^{2 n}}+\int_{0}^{t}\left\|\partial_{x} g * F(u, \rho)(\tau)\right\|_{L^{2 n}} \mathrm{~d} \tau\right) e^{\lambda_{1} t} .
$$

Taking the limit as $n \rightarrow \infty$ in (2.5), one gets

$$
\|u(t)\|_{L^{\infty}} \leq\left(\left\|u_{0}\right\|_{L^{\infty}}+\int_{0}^{t}\left\|\partial_{x} g * F(u, \rho)(\tau)\right\|_{L^{\infty}} \mathrm{d} \tau\right) e^{\lambda_{1} t} .
$$

Differentiating the first equation in (1.6) in the variable $x$ yields

$$
u_{x t}+u u_{x x}+u_{x}^{2}+\lambda_{1} u_{x}+\partial_{x}^{2} g * F(u, \rho)=0 .
$$

Multiplying (2.7) by $u_{x}^{2 n-1}$ with $n \in \mathbb{N}^{*}$, integrating the resultant equation with respect to $x$ and using

$$
\int_{\mathbb{R}}\left(u u_{x x} u_{x}^{2 n-1}+u_{x}^{2} u_{x}^{2 n-1}\right) \mathrm{d} x=\left(1-\frac{1}{2 n}\right) \int_{\mathbb{R}} u_{x}^{2 n+1} \mathrm{~d} x \leq\left\|u_{x}\right\|_{L^{\infty}}\left\|u_{x}\right\|_{L^{2 n}}^{2 n}
$$

and

$$
\int_{\mathbb{R}}\left[\partial_{x}^{2} g * F(u, \rho)\right] u_{x}^{2 n-1} \mathrm{~d} x \leq\left\|\partial_{x}^{2} g * F(u, \rho)\right\|_{L^{2 n}}\left\|u_{x}\right\|_{L^{2 n}}^{2 n-1},
$$

we have

$$
\frac{\mathrm{d}}{\mathrm{d} t}\left\|u_{x}\right\|_{L^{2 n}} \leq\left(\left\|u_{x}\right\|_{L^{\infty}}+\lambda_{1}\right)\left\|u_{x}\right\|_{L^{2 n}}+\left\|\partial_{x}^{2} g * F(u, \rho)\right\|_{L^{2 n}} .
$$


We obtain

$$
\left\|u_{x}(t)\right\|_{L^{\infty}} \leq\left[\left\|u_{0 x}\right\|_{L^{\infty}}+\int_{0}^{t}\left\|\partial_{x}^{2} g * F(u, \rho)(\tau)\right\|_{L^{\infty}} \mathrm{d} \tau\right] e^{\left(M+\lambda_{1}\right) t} .
$$

We introduce the weight function $\varphi_{N}(x)$ which is independent on $t$

$$
\varphi_{N}(x)= \begin{cases}1, & x \leq 0, \\ e^{\theta x}, & x \in(0, N), \\ e^{\theta N}, & x \geq N,\end{cases}
$$

where $N \in \mathbb{N}^{*}$. It follows $0 \leq\left(\varphi_{N}(x)\right)_{x} \leq \varphi_{N}(x)$ a.e. $x \in \mathbb{R}$. Multiplying the first equation in system (1.6) and (2.7) by $\varphi_{N}(x)$, we obtain

$$
\begin{aligned}
& \partial_{t}\left(u \varphi_{N}\right)+u \varphi_{N} u_{x}+\lambda_{1} u \varphi_{N}+\varphi_{N} \partial_{x} g * F(u, \rho)=0, \\
& \partial_{t}\left(u_{x} \varphi_{N}\right)+u u_{x x} \varphi_{N}+u_{x} \varphi_{N} u_{x}+\lambda_{1} u_{x} \varphi_{N}+\varphi_{N} \partial_{x}^{2} g * F(u, \rho)=0 .
\end{aligned}
$$

Multiplying (2.10) by $\left(u \varphi_{N}\right)^{2 n-1}$ and (2.11) by $\left(u_{x} \varphi_{N}\right)^{2 n-1}$, respectively, and integrating the resultant equation with respect to $x$, we also note

$$
\begin{aligned}
\left|\int_{\mathbb{R}} u u_{x x} \varphi_{N}\left(u_{x} \varphi_{N}\right)^{2 n-1} \mathrm{~d} x\right| & =\left|\int_{\mathbb{R}} u\left(u_{x} \varphi_{N}\right)^{2 n-1}\left[\partial_{x}\left(u_{x} \varphi_{N}\right)-u_{x}\left(\varphi_{N}\right)_{x}\right] \mathrm{d} x\right| \\
& \leq\left|\frac{1}{2 n} \int_{\mathbb{R}} u_{x}\left(u_{x} \varphi_{N}\right)^{2 n} \mathrm{~d} x\right|+\left|\int_{\mathbb{R}} u u_{x} \varphi_{N}\left(u_{x} \varphi_{N}\right)^{2 n-1} \mathrm{~d} x\right| \\
& \leq\left(\|u(t)\|_{L^{\infty}}+\left\|u_{x}(t)\right\|_{L^{\infty}}\right)\left\|u_{x} \varphi_{N}\right\|_{L^{2 n}}^{2 n} .
\end{aligned}
$$

As in the weightless case, we estimate $u \varphi_{N}$ and $u_{x} \varphi_{N}$ step by step as the previous estimates for $u$ and $u_{x}$. Thus

$$
\begin{aligned}
& \left\|u \varphi_{N}\right\|_{L^{\infty}}+\left\|u_{x} \varphi_{N}\right\|_{L^{\infty}} \\
& \leq e^{\left(4 M+2 \lambda_{1}\right) t}\left(\left\|u_{0} \varphi_{N}\right\|_{L^{\infty}}+\left\|u_{0 x} \varphi_{N}\right\|_{L^{\infty}}\right) \\
& \quad+e^{\left(4 M+2 \lambda_{1}\right) t} \int_{0}^{t}\left(\left\|\varphi_{N} \partial_{x} g * F(u, \rho)\right\|_{L^{\infty}}+\left\|\varphi_{N} \partial_{x}^{2} g * F(u, \rho)\right\|_{L^{\infty}}\right) \mathrm{d} \tau .
\end{aligned}
$$

Multiplying the second equation in system (1.6) by $\varphi_{N}$, one deduces

$$
\left(\rho \varphi_{N}\right)_{t}+u \rho_{x} \varphi_{N}+2 u_{x} \rho \varphi_{N}+\lambda \rho \varphi_{N}=0 .
$$

Multiplying (2.13) by $\left(\rho \varphi_{N}\right)^{2 n-1}$, integrating the resultant equation with respect to $x$ and using

$$
\begin{aligned}
\left|\int_{\mathbb{R}} u \rho_{x} \varphi_{N}\left(\rho \varphi_{N}\right)^{2 n-1} \mathrm{~d} x\right| & =\left|\int_{\mathbb{R}} u\left(\rho \varphi_{N}\right)^{2 n-1}\left[\partial_{x}\left(\rho \varphi_{N}\right)-\rho\left(\varphi_{N}\right)_{x}\right] \mathrm{d} x\right| \\
& \leq\left|\frac{1}{2 n} \int_{\mathbb{R}} u_{x}\left(\rho \varphi_{N}\right)^{2 n} \mathrm{~d} x\right|+\left|\int_{\mathbb{R}} u \rho \varphi_{N}\left(\rho \varphi_{N}\right)^{2 n-1} \mathrm{~d} x\right| \\
& \leq\left(\|u(t)\|_{L^{\infty}}+\left\|u_{x}(t)\right\|_{L^{\infty}}\right)\left\|\rho \varphi_{N}\right\|_{L^{2 n}}^{2 n}, \\
\int_{\mathbb{R}} 2 u_{x} \rho \varphi_{N}\left(\rho \varphi_{N}\right)^{2 n-1} \mathrm{~d} x & \leq 2\left\|u_{x}(t)\right\|_{L^{\infty}}\left\|\rho \varphi_{N}\right\|_{L^{2 n}}^{2 n},
\end{aligned}
$$


we have

$$
\frac{\mathrm{d}}{\mathrm{d} t}\left\|\rho \varphi_{N}\right\|_{L^{2 n}} \leq\left(3\left\|u_{x}\right\|_{L^{\infty}}+\|u(t)\|_{L^{\infty}}+\lambda\right)\left\|\rho \varphi_{N}\right\|_{L^{2 n}}
$$

Applying the Gronwall inequality and the Sobolev embedding theorem yields

$$
\left\|\rho \varphi_{N}\right\|_{L^{2 n}} \leq e^{(4 M+\lambda) t}\left\|\rho_{0} \varphi_{N}\right\|_{L^{2 n}}
$$

Taking the limit as $n \rightarrow \infty$, one obtains

$$
\left\|\rho \varphi_{N}\right\|_{L^{\infty}} \leq e^{(4 M+\lambda) t}\left\|\rho_{0} \varphi_{N}\right\|_{L^{\infty}}
$$

There exists $c_{1}>0$ which depends on $\theta \in(0,1)$, such that for all $N \in \mathbb{N}^{*}$

$$
\varphi_{N}(x) \int_{\mathbb{R}} e^{-|x-y|} \frac{1}{\varphi_{N}(y)} \mathrm{d} y \leq c_{1} .
$$

Thus

$$
\begin{aligned}
& \left|\varphi_{N} \partial_{x} g * F(u, \rho)\right| \\
& \quad \leq\left|\frac{1}{2} \varphi_{N}(x) \int_{\mathbb{R}} \operatorname{sgn}(x-y) e^{-|x-y|}\left(\frac{3}{2} u^{2}+\frac{c}{2} \rho^{2}\right) \mathrm{d} y\right| \\
& \quad \leq \frac{1}{2} \varphi_{N}(x)\left|\int_{\mathbb{R}} e^{-|x-y|} \frac{1}{\varphi_{N}(y)} \varphi_{N}(y)\left(\frac{3}{2} u^{2}+\frac{c}{2} \rho^{2}\right) \mathrm{d} y\right| \\
& \quad \leq \frac{(3+c) c_{1}}{4}\left(\|u\|_{L^{\infty}}\left\|u \varphi_{N}\right\|_{L^{\infty}}+\|\rho\|_{L^{\infty}}\left\|\rho \varphi_{N}\right\|_{L^{\infty}}\right) .
\end{aligned}
$$

Using $\partial_{x}^{2} g * f=g * f-f$ for all $f$, we have

$$
\begin{aligned}
& \left|\varphi_{N} \partial_{x}^{2} g * F(u, \rho)\right| \\
& \quad=\left|\frac{1}{2} \varphi_{N}(x) \int_{\mathbb{R}} e^{-|x-y|}\left(\frac{3}{2} u^{2}+\frac{c}{2} \rho^{2}\right) \mathrm{d} y-\varphi_{N}(x)\left(\frac{3}{2} u^{2}+\frac{c}{2} \rho^{2}\right)\right| \\
& \quad \leq \frac{1}{2} \varphi_{N}\left|\int_{\mathbb{R}} e^{-|x-y|} \frac{1}{\varphi_{N}(y)} \varphi_{N}(y)\left(\frac{3}{2} u^{2}+\frac{c}{2} \rho^{2}\right) \mathrm{d} y\right|+\left|\varphi_{N}(x)\left(\frac{3}{2} u^{2}+\frac{c}{2} \rho^{2}\right)\right| \\
& \quad \leq \frac{(3+c)\left(c_{1}+2\right)}{4}\left(\|u\|_{L^{\infty}}\left\|u \varphi_{N}\right\|_{L^{\infty}}+\|\rho\|_{L^{\infty}}\left\|\rho \varphi_{N}\right\|_{L^{\infty}}\right) .
\end{aligned}
$$

Plugging (2.15), (2.16) into (2.12) and using (2.14), there exists $C_{1}>0$ such that

$$
\begin{aligned}
& \left\|u \varphi_{N}\right\|_{L^{\infty}}+\left\|u_{x} \varphi_{N}\right\|_{L^{\infty}}+\left\|\rho \varphi_{N}\right\|_{L^{\infty}} \\
& \leq C_{1}\left(\left\|u_{0} \varphi_{N}\right\|_{L^{\infty}}+\left\|u_{0 x} \varphi_{N}\right\|_{L^{\infty}}+\left\|\rho_{0} \varphi_{N}\right\|_{L^{\infty}}\right) \\
& \quad+C_{1} \int_{0}^{t}\left(\|u\|_{L^{\infty}}+\left\|u_{x}\right\|_{L^{\infty}}+\|\rho\|_{L^{\infty}}\right) \\
& \quad \times\left(\left\|u \varphi_{N}\right\|_{L^{\infty}}+\left\|u_{x} \varphi_{N}\right\|_{L^{\infty}}+\left\|\rho \varphi_{N}\right\|_{L^{\infty}}\right) \mathrm{d} \tau .
\end{aligned}
$$


Using the Gronwall inequality, one deduces that for all $N \in \mathbb{N}^{*}$ and $t \in[0, T]$

$$
\begin{aligned}
& \left\|u \varphi_{N}\right\|_{L^{\infty}}+\left\|u_{x} \varphi_{N}\right\|_{L^{\infty}}+\left\|\rho \varphi_{N}\right\|_{L^{\infty}} \\
& \leq C\left(\left\|u_{0} \varphi_{N}\right\|_{L^{\infty}}+\left\|u_{0 x} \varphi_{N}\right\|_{L^{\infty}}+\left\|\rho_{0} \varphi_{N}\right\|_{L^{\infty}}\right) \\
& \leq C\left(\left\|e^{\theta x} u_{0}\right\|_{L^{\infty}}+\left\|e^{\theta x} u_{0 x}\right\|_{L^{\infty}}+\left\|e^{\theta x} \rho_{0}\right\|_{L^{\infty}}\right) .
\end{aligned}
$$

Finally, taking the limit as $N \rightarrow \infty$, one obtains

$$
\begin{aligned}
& \sup _{t \in[0, T]}\left(\left\|e^{\theta x} u\right\|_{L^{\infty}}+\left\|e^{\theta x} u_{x}\right\|_{L^{\infty}}+\left\|e^{\theta x} \rho\right\|_{L^{\infty}}\right) \\
& \quad \leq C\left(\left\|e^{\theta x} u_{0}\right\|_{L^{\infty}}+\left\|e^{\theta x} u_{0 x}\right\|_{L^{\infty}}+\left\|e^{\theta x} \rho_{0}\right\|_{L^{\infty}}\right) .
\end{aligned}
$$

Thus

$$
|u(t, x)|,\left|\partial_{x} u(t, x)\right|,|\rho(t, x)| \sim O\left(e^{-\theta x}\right) \quad \text { as } x \rightarrow \infty
$$

uniformly on the interval $[0, T]$. This completes the proof of Theorem 1.2.

\section{Proofs of Theorems 1.3 and 1.4}

\subsection{Proof of Theorem 1.3}

(1) For $c>0$, integrating the first equation in (1.6) over the interval $\left[0, t_{1}\right]$, one has

$$
\begin{aligned}
& u\left(t_{1}, x\right)-u(0, x)+\int_{0}^{t_{1}} u u_{x}(\tau, x) \mathrm{d} \tau+\lambda_{1} \int_{0}^{t_{1}} u(\tau, x) \mathrm{d} \tau \\
& =-\int_{0}^{t_{1}} \partial_{x} g *\left(\frac{3}{2} u^{2}+\frac{c}{2} \rho^{2}\right) \mathrm{d} \tau
\end{aligned}
$$

From the assumption in Theorem 1.3, one deduces

$$
u\left(t_{1}, x\right)-u(0, x) \sim o\left(e^{-x}\right) \quad \text { as } x \rightarrow \infty
$$

It follows from Theorem 1.2 that

$$
\begin{aligned}
& \int_{0}^{t_{1}} u u_{x}(\tau, x) \mathrm{d} \tau \sim O\left(e^{-2 \theta x}\right) \quad \text { as } x \rightarrow \infty ; \\
& \int_{0}^{t_{1}} u(\tau, x) \mathrm{d} \tau \sim O\left(e^{-\theta x}\right) \quad \text { as } x \rightarrow \infty .
\end{aligned}
$$

For $\theta \in\left(\frac{1}{2}, 1\right)$, we have

$$
\int_{0}^{t_{1}} u u_{x}(\tau, x) \mathrm{d} \tau \sim o\left(e^{-x}\right) \quad \text { as } x \rightarrow \infty
$$

For the right side in (3.1), we have

$$
\int_{0}^{t_{1}} \partial_{x} g *\left(\frac{3}{2} u^{2}+\frac{c}{2} \rho^{2}\right) \mathrm{d} \tau=\partial_{x} g * \int_{0}^{t_{1}}\left(\frac{3}{2} u^{2}+\frac{c}{2} \rho^{2}\right) \mathrm{d} \tau=\partial_{x} g * p(x),
$$


where $p(x)=\int_{0}^{t_{1}}\left(\frac{3}{2} u^{2}+\frac{c}{2} \rho^{2}\right) \mathrm{d} \tau$. From Theorem 1.2, one has

$$
0 \leq p(x) \sim O\left(e^{-2 \theta x}\right) \text {, thus } p(x) \sim o\left(e^{-x}\right) \quad \text { as } x \rightarrow \infty
$$

Then

$$
\begin{aligned}
\partial_{x} g * p(x) & =-\frac{1}{2} \int_{\mathbb{R}} \operatorname{sgn}(x-y) e^{-|x-y|} p(y) \mathrm{d} y \\
& =-\frac{1}{2} e^{-x} \int_{-\infty}^{x} e^{y} p(y) \mathrm{d} y+\frac{1}{2} e^{x} \int_{x}^{\infty} e^{-y} p(y) \mathrm{d} y .
\end{aligned}
$$

Noting $e^{x} \int_{x}^{\infty} e^{-y} p(y) \mathrm{d} y=o(1) e^{x} \int_{x}^{\infty} e^{-2 y} \mathrm{~d} y=o(1) e^{-x} \sim o\left(e^{-x}\right)$, if there is at least one of the equalities $u(t, x) \neq 0$ and $\rho(t, x) \neq 0$ is valid, we have $p(x) \neq 0$. Then there exists $c_{2}>0$ such that

$$
\int_{-\infty}^{x} e^{y} p(y) \mathrm{d} y \geq c_{2} \quad \text { for } x \gg 1
$$

Thus

$$
-\partial_{x} g * p(x) \geq \frac{c_{2}}{2} e^{-x} \quad \text { for } x \gg 1
$$

which combined with the above estimates yields a contradiction. We obtain $p(x)=0$. Consequently, $u=0, \rho=0$.

(2) For $c=0$, similar to the case $c>0$, one deduces $u=0$. Inserting $u=0$ into the second equation in (1.1), one derives

$$
\rho_{t}=-\lambda \rho
$$

From (3.5), we have $\rho(t, x)=\rho_{0}(x) e^{-\lambda t}$. This completes the proof of case (2) in Theorem 1.3.

\subsection{Proof of Theorem 1.4}

For $\lambda_{1}=0$, integrating the first equation in system (1.6) on the interval $[0, t]$, one obtains

$$
u(t, x)-u(0, x)+\int_{0}^{t} u u_{x}(\tau, x) \mathrm{d} \tau=-\int_{0}^{t} \partial_{x} g *\left(\frac{3}{2} u^{2}+\frac{c}{2} \rho^{2}\right) \mathrm{d} \tau
$$

From the assumption in Theorem $1.4 u(0, x) \sim O\left(e^{-x}\right)$ as $x \rightarrow \infty$ and Theorem 1.2, one deduces

$$
\int_{0}^{t} u u_{x}(\tau, x) \mathrm{d} \tau \sim O\left(e^{-2 \theta x}\right) \quad \text { as } x \rightarrow \infty
$$

For $\theta \in\left(\frac{1}{2}, 1\right)$, then

$$
\int_{0}^{t} u u_{x}(\tau, x) \mathrm{d} \tau \sim o\left(e^{-x}\right) \quad \text { as } x \rightarrow \infty
$$

For the right side in (3.6), firstly, we have

$$
\frac{3}{2} \int_{0}^{t} \partial_{x} g * u^{2}(\tau, x) \mathrm{d} \tau=\frac{3}{2} \partial_{x} g * \int_{0}^{t} u^{2}(\tau, x) \mathrm{d} \tau=\frac{3}{2} \partial_{x} g * p_{1}(x),
$$


where $p_{1}(x)=\int_{0}^{t} u^{2}(\tau, x) \mathrm{d} \tau$. Using Theorem 1.2, we obtain

$$
0 \leq p_{1}(x) \sim O\left(e^{-2 \theta x}\right) \quad \text { as } x \rightarrow \infty .
$$

Thus

$$
\begin{aligned}
\partial_{x} g * p_{1}(x) & =-\frac{1}{2} \int_{\mathbb{R}} \operatorname{sgn}(x-y) e^{-|x-y|} p_{1}(y) \mathrm{d} y \\
& =-\frac{1}{2} e^{-x} \int_{-\infty}^{x} e^{y} p_{1}(y) \mathrm{d} y+\frac{1}{2} e^{x} \int_{x}^{\infty} e^{-y} p_{1}(y) \mathrm{d} y .
\end{aligned}
$$

Noting

$$
e^{x} \int_{x}^{\infty} e^{-y} p_{1}(y) \mathrm{d} y \sim O\left(e^{-2 \theta x}\right) \sim o\left(e^{-x}\right) \quad \text { as } x \rightarrow \infty
$$

and

$$
-\frac{1}{2} e^{-x} \int_{-\infty}^{x} e^{y} p_{1}(y) \mathrm{d} y \leq-\frac{c_{0}}{2} e^{-x} \quad \text { for } x \gg 1,
$$

we have

$$
\frac{3}{2} \int_{0}^{t} \partial_{x} g * u^{2}(\tau, x) \mathrm{d} \tau \sim O\left(e^{-x}\right) \quad \text { as } x \rightarrow \infty .
$$

Similarly, we have

$$
\frac{3}{2} \int_{0}^{t} \partial_{x} g * \rho^{2}(\tau, x) \mathrm{d} \tau \sim O\left(e^{-x}\right) \quad \text { as } x \rightarrow \infty .
$$

Then $u(t, x) \sim O\left(e^{-x}\right)$ as $x \rightarrow \infty$. From Theorem 1.2, if $\rho_{0}(x) \sim O\left(e^{-\theta x}\right)$ as $x \rightarrow \infty$, we have $\rho(t, x) \sim O\left(e^{\theta x}\right)$ as $x \rightarrow \infty$. This completes the proof of Theorem 1.4.

\section{Proofs of Theorems 1.5 and 1.6}

\subsection{Proof of Theorem 1.5}

From the proof of Theorem 1.2, here we need to differentiate the second equation in (1.6) with variable $x$, and one has

$$
\rho_{x t}+3 u_{x} \rho_{x}+u \rho_{x x}+2 u_{x x} \rho+\lambda \rho_{x}=0
$$

Multiplying (4.1) by $\rho_{x}^{2 n-1}$ and integrating the resultant equation with respect to $x$, we also note

$$
\begin{aligned}
& \int_{\mathbb{R}} \rho_{x t} \rho_{x}^{2 n-1} \mathrm{~d} x=\left\|\rho_{x}\right\|_{L^{2 n}}^{2 n-1} \frac{\mathrm{d}}{\mathrm{d} t}\left\|\rho_{x}\right\|_{L^{2 n}}, \\
& \int_{\mathbb{R}}\left(3 u_{x} \rho_{x}+u \rho_{x x}\right) \rho_{x}^{2 n-1} \mathrm{~d} x=\left(3-\frac{1}{2 n}\right) \int_{\mathbb{R}} u_{x} \rho_{x}^{2 n} \mathrm{~d} x \leq 3\left\|u_{x}\right\|_{L^{\infty}}\left\|\rho_{x}\right\|_{L^{2 n}}^{2 n}, \\
& 2 \int_{\mathbb{R}} u_{x x} \rho \rho_{x}^{2 n-1} \mathrm{~d} x \leq 2\left\|u_{x x} \rho\right\|_{L^{2 n}}\left\|\rho_{x}\right\|_{L^{2 n}}^{2 n-1} \leq 2\left\|u_{x x}\right\|_{L^{\infty}}\|\rho\|_{L^{2 n}}\left\|\rho_{x}\right\|_{L^{2 n}}^{2 n-1}, \\
& \lambda \int_{\mathbb{R}} \rho_{x} \rho_{x}^{2 n-1} \mathrm{~d} x=\lambda\left\|\rho_{x}\right\|_{L^{2 n}}^{2 n} .
\end{aligned}
$$


Thus, we obtain

$$
\frac{\mathrm{d}}{\mathrm{d} t}\left\|\rho_{x}\right\|_{L^{2 n}} \leq\left(3\left\|u_{x}\right\|_{L^{\infty}}+\lambda\right)\left\|\rho_{x}\right\|_{L^{2 n}}+2\left\|u_{x x}\right\|_{L^{\infty}}\|\rho\|_{L^{2 n}}
$$

Taking the limit as $n \rightarrow \infty$ and applying the Gronwall inequality yield

$$
\left\|\rho_{x}\right\|_{L^{\infty}} \leq\left[\left\|\rho_{0 x}\right\|_{L^{\infty}}+2 M \int_{0}^{t}\|\rho\|_{L^{\infty}} \mathrm{d} \tau\right] e^{(3 M+\lambda) t}
$$

In order to obtain the estimates for $\left\|\rho_{x} \varphi_{N}\right\|_{L^{\infty}}$, we multiply (4.1) with the weight function $\varphi_{N}(x)$, then

$$
\rho_{x t} \varphi_{N}+3 u_{x} \rho_{x} \varphi_{N}+u \rho_{x x} \varphi_{N}+2 u_{x x} \rho \varphi_{N}+\lambda \rho_{x} \varphi_{N}=0
$$

Multiplying (4.4) with $\left(\rho_{x} \varphi_{N}\right)^{2 n-1}$ and integrating the resultant equation with respect to $x$, we note

$$
\begin{aligned}
& \int_{\mathbb{R}} \rho_{x t} \varphi_{N}\left(\rho_{x} \varphi_{N}\right)^{2 n-1} \mathrm{~d} x=\left\|\rho_{x} \varphi_{N}\right\|_{L^{2 n}}^{2 n-1} \frac{\mathrm{d}}{\mathrm{d} t}\left\|\rho_{x} \varphi_{N}\right\|_{L^{2 n}}, \\
& \int_{\mathbb{R}}\left(3 u_{x} \rho_{x} \varphi_{N}\right)\left(\rho_{x} \varphi_{N}\right)^{2 n-1} \mathrm{~d} x \leq 3\left\|u_{x}\right\|_{L^{\infty}}\left\|\rho_{x} \varphi_{N}\right\|_{L^{2 n}}^{2 n}, \\
& \int_{\mathbb{R}}\left(u \rho_{x x} \varphi_{N}\right)\left(\rho_{x} \varphi_{N}\right)^{2 n-1} \mathrm{~d} x \leq \int_{\mathbb{R}} u\left(\rho_{x} \varphi_{N}\right)^{2 n-1}\left[\partial_{x}\left(\rho_{x} \varphi_{N}\right)-\rho_{x}\left(\varphi_{N}\right)_{x}\right] \mathrm{d} x \\
& \leq\left|\int_{\mathbb{R}} u_{x}\left(\rho_{x} \varphi_{N}\right)^{2 n} \mathrm{~d} x\right|+\left|\int_{\mathbb{R}} u\left(\rho_{x} \varphi_{N}\right)^{2 n} \mathrm{~d} x\right| \\
& \leq\left(\|u\|_{L^{\infty}}+\left\|u_{x}\right\|_{L^{\infty}}\right)\left\|\rho_{x} \varphi_{N}\right\|_{L^{2 n}}^{2 n}, \\
& 2 \int_{\mathbb{R}} u_{x x} \rho \varphi_{N}\left(\rho_{x} \varphi_{N}\right)^{2 n-1} \mathrm{~d} x \leq 2\left\|u_{x x}\right\|_{L^{\infty}}\left\|\rho \varphi_{N}\right\|_{L^{2 n}}\left\|\rho_{x} \varphi_{N}\right\|_{L^{2 n}}^{2 n-1}, \\
& \lambda \int_{\mathbb{R}} \rho_{x} \varphi_{N}\left(\rho_{x} \varphi_{N}\right)^{2 n-1} \mathrm{~d} x=\lambda\left\|\rho_{x} \varphi_{N}\right\|_{L^{2 n}}^{2 n} .
\end{aligned}
$$

Hence, we have

$$
\begin{aligned}
& \frac{\mathrm{d}}{\mathrm{d} t}\left\|\rho_{x} \varphi_{N}\right\|_{L^{2 n}} \\
& \quad \leq\left(4\left\|u_{x}\right\|_{L^{\infty}}+\|u\|_{L^{\infty}}+\lambda\right)\left\|\rho_{x} \varphi_{N}\right\|_{L^{2 n}}+2\left\|u_{x x}\right\|_{L^{\infty}}\left\|\rho \varphi_{N}\right\|_{L^{2 n}} .
\end{aligned}
$$

Taking the limit as $n \rightarrow \infty$ and using the Gronwall inequality, one obtains

$$
\left\|\rho_{x} \varphi_{N}\right\|_{L^{\infty}} \leq\left(\left\|\rho_{0 x} \varphi_{N}\right\|_{L^{\infty}}+2 M \int_{0}^{t}\left\|\rho \varphi_{N}\right\|_{L^{\infty}} \mathrm{d} \tau\right) e^{(5 M+\lambda) t}
$$

From (4.6) and (2.17), one deduces that there exists $C_{2}$ such that

$$
Y(t) \leq C_{2} Y_{0}+C_{2} \int_{0}^{t} y(\tau) Y(\tau) \mathrm{d} \tau
$$


where

$$
\begin{aligned}
& Y(t)=\left\|u \varphi_{N}\right\|_{L^{\infty}}+\left\|u_{x} \varphi_{N}\right\|_{L^{\infty}}+\left\|\rho \varphi_{N}\right\|_{L^{\infty}}+\left\|\rho_{x} \varphi_{N}\right\|_{L^{\infty}}, \\
& y(t)=\|u\|_{L^{\infty}}+\left\|u_{x}\right\|_{L^{\infty}}+\left\|u_{x x}\right\|_{L^{\infty}}+\|\rho\|_{L^{\infty}}+\left\|\rho_{x}\right\|_{L^{\infty}}+1 .
\end{aligned}
$$

Applying the Gronwall inequality to (4.7), for all $N \in \mathbb{N}^{*}$ and $t \in[0, T]$, one has

$$
Y(t) \leq C Y_{0} \leq C\left(\left\|u_{0} e^{\theta x}\right\|_{L^{\infty}}+\left\|u_{0 x} e^{\theta x}\right\|_{L^{\infty}}+\left\|\rho_{0} e^{\theta x}\right\|_{L^{\infty}}+\left\|\rho_{0 x} e^{\theta x}\right\|_{L^{\infty}}\right) .
$$

Now taking the limit as $N \rightarrow \infty$ in (4.8), one obtains

$$
\begin{aligned}
& \sup _{t \in[0, T]}\left(\left\|u(t, x) e^{\theta x}\right\|_{L^{\infty}}+\left\|u_{x}(t, x) e^{\theta x}\right\|_{L^{\infty}}+\left\|\rho(t, x) e^{\theta x}\right\|_{L^{\infty}}+\left\|\rho_{x}(t, x) e^{\theta x}\right\|_{L^{\infty}}\right) \\
& \quad \leq C\left(\left\|u_{0} e^{\theta x}\right\|_{L^{\infty}}+\left\|u_{0 x} e^{\theta x}\right\|_{L^{\infty}}+\left\|\rho_{0} e^{\theta x}\right\|_{L^{\infty}}+\left\|\rho_{0 x} e^{\theta x}\right\|_{L^{\infty}}\right) .
\end{aligned}
$$

Using the assumption in Theorem 1.5, we complete the proof.

\subsection{Proof of Theorem 1.6}

The proof of Theorem 1.6 is similar to the proof of Theorem 1.3, here we omit it.

\section{Competing interests}

The authors declare that they have no competing interests.

\section{Authors' contributions}

All authors contributed to each part of this study equally and approved the final version of the manuscript.

\section{Author details}

'School of Mathematics, Southwest Jiaotong University, Chengdu, 610031, China. ${ }^{2}$ Department of Mathematics, Southwestern University of Finance and Economics, Chengdu, 611130, China.

\section{Acknowledgements}

The authors would like to express sincere gratitude to the anonymous referees for a number of valuable comments and suggestions. This work was partially supported by National Natural Science Foundation of P.R. China (71003082) and Fundamental Research Funds for the Central Universities (SWJTU12CX061 and SWJTU09ZT36).

Received: 29 March 2014 Accepted: 28 April 2014 Published: 09 May 2014

\section{References}

1. Degasperis, A, Procesi, M: Asymptotic integrability. In: Symmetry and Perturbation Theory, pp. 23-37. World Scientific, Singapore (1999)

2. Escher, J, Liu, Y, Yin, Z: Global weak solutions and blow-up structure for the Degasperis-Procesi equation. J. Funct. Anal. 241, 457-485 (2006)

3. Liu, Y, Yin, Z: Global existence and blow-up phenomena for the Degasperis-Procesi equation. Commun. Math. Phys. $267,801-820(2006)$

4. Yin, Z: On the Cauchy problem for an integrable equation with peakon solutions. III. J. Math. 47, $649-666$ (2003)

5. Yin, Z: Global existence for a new periodic integrable equation. J. Math. Anal. Appl. 283, 129-139 (2003)

6. Yin, Z: Global solutions to a new integrable equation with peakons. Indiana Univ. Math. J. 53, 1189-1210 (2004)

7. Yin, Z: Global weak solutions to a new periodic integrable equation with peakon solutions. J. Funct. Anal. 212, 182-194 (2004)

8. Wu, S, Yin, Z: Blow-up phenomena and decay for the periodic Degasperis-Procesi equation with weak dissipation. J. Nonlinear Math. Phys. 15, 28-49 (2008)

9. Guo, Y, Lai, S, Wang, Y: Global weak solutions to the weakly dissipative Degasperis-Procesi equation. Nonlinear Anal. 74, 4961-4973 (2011)

10. Guo, Z: Some properties of solutions to the weakly dissipative Degasperis-Procesi equation. J. Differ. Equ. 246 4332-4344 (2009)

11. Yan, K, Yin, Z: On the Cauchy problem for a 2-component Degasperis-Procesi system. J. Differ. Equ. 252, 2131-2159 (2012)

12. Zhou, J, Tian, L, Fan, X: Soliton, kink and antikink solutions of a 2-component of the Degasperis-Procesi equation. Nonlinear Anal., Real World Appl. 11, 2529-2536 (2012) 
13. $\mathrm{Fu}, \mathrm{Y}, \mathrm{Qu}, \mathrm{C}$ : Unique continuation and persistence properties of solutions of the 2-component Degasperis-Procesi equations. Acta Math. Sci. 32, 652-662 (2012)

14. Jin, L, Guo, Z: On a 2-component Degasperis-Procesi shallow water system. Nonlinear Anal., Real World Appl. 11, 4164-4173 (2010)

15. Yu, L, Tian, L: Loop solutions, breaking kink wave solutions, solitary wave solutions and periodic wave solutions for the 2-component Degasperis-Procesi equation. Nonlinear Anal., Real World Appl. 15, 140-148 (2014)

16. Manwai, Y: Self-similar blow-up solutions to the 2-component Degasperis-Procesi shallow water system. Commun. Nonlinear Sci. Numer. Simul. 16, 3463-3469 (2011)

17. Hu, Q: Global existence and blow-up phenomena for a weakly dissipative 2-component Camassa-Holm system. Appl. Anal. 92, 398-410 (2013)

18. Hu, Q: Global existence and blow-up phenomena for a weakly dissipative periodic 2-component Camassa-Holm system. J. Math. Phys. 52, 103701 (2011)

19. Jin, L, Guo, Z: A note on a modified 2-component Camassa-Holm system. Nonlinear Anal., Real World Appl. 13 887-892 (2012)

20. Zhu, M: Blow-up, global existence and persistence properties for the coupled Camassa-Holm equations. Math. Phys. Anal. Geom. 14, 197-209 (2011)

21. Guo, Z: Asymptotic profiles of solutions to the 2-component Camassa-Holm system. Nonlinear Anal. 75, 1-6 (2012)

22. Guo, Z, Ni, L: Persistence properties and unique continuation of solutions to a 2-component Camassa-Holm equation. Math. Phys. Anal. Geom. 14, 101-114 (2011)

23. Constantin, A, Ivanov, R: On an integrable 2-component Camassa-Holm shallow water system. Phys. Lett. A 372 7129-7132 (2008)

24. Gui, G, Liu, Y: On the global existence and wave-breaking criteria for the 2-component Camassa-Holm system. J. Funct. Anal. 258, 4251-4278 (2010)

25. Gui, G, Liu, Y: On the Cauchy problem for the 2-component Camassa-Holm system. Math. Z. 268, 45-66 (2011)

26. Ai, X, Gui, G: Global well-posedness for the Cauchy problem of the viscous Degasperis-Procesi equation. J. Math. Anal. Appl. 361, 457-465 (2010)

27. Chen, W, Tian, L, Deng, X, Zhang, J: Wave breaking for a generalized weakly dissipative 2-component Camassa-Holm system. J. Math. Anal. Appl. 400, 406-417 (2013)

28. Jin, L, Jiang, Z: Wave breaking of an integrable Camassa-Holm system with 2-component. Nonlinear Anal. 95, 107-116 (2014)

29. Lenells, J, Wunsch, M: On the weakly dissipative Camassa-Holm, Degasperis-Procesi, and Novikov equations. J. Differ. Equ. 255, 441-448 (2013)

30. Zhu, M: On a shallow water equation perturbed in Schwartz class. Math. Phys. Anal. Geom. (2012). doi:10.1007/s11040-012-9112-z

31. Zhu, M, Jiang, Z: Some properties of solutions to the weakly dissipative $b$-family equation. Nonlinear Anal., Real World Appl. 13, 158-167 (2012)

32. Ni, L, Zhou, Y: Well-posedness and persistence properties for the Novikov equation. J. Differ. Equ. 250, 3002-3021 (2011)

33. Zong, X: Properties of the solutions to the 2-component $b$-family systems. Nonlinear Anal. 75, 6250-6259 (2012)

34. Zhou, S, Mu, C: The properties of solutions for a generalized $b$-family equation with peakons. J. Nonlinear Sci. 23 863-889 (2013)

35. Himonas, A, Misiolek, G, Ponce, G, Zhou, Y: Persistence properties and unique continuation of solutions to the Camassa-Holm equation. Math. Phys. Anal. Geom. 271, 511-522 (2007)

36. Danchin, R: A few remarks on the Camassa-Holm equation. Differ. Integral Equ. 14, 953-988 (2001)

37. Danchin, R: A note on well-posedness for Camassa-Holm equation. J. Differ. Equ. 192, 429-444 (2003)

38. Danchin, R: Fourier analysis methods for PDEs. Lecture Notes, 14 Nov. Preprint (2005)

39. Bahouri, H, Chemin, J, Danchin, R: Fourier Analysis and Nonlinear Partial Differential Equations. Grun. der Math. Wiss. Springer, Berlin (2010)

10.1186/1687-2770-2014-108

Cite this article as: Ming et al.: Persistence properties of solutions to the dissipative 2-component

Degasperis-Procesi system. Boundary Value Problems 2014, 2014:108

\section{Submit your manuscript to a SpringerOpen ${ }^{\ominus}$ journal and benefit from:}

- Convenient online submission

Rigorous peer review

- Immediate publication on acceptance

Open access: articles freely available online

- High visibility within the field

- Retaining the copyright to your article

Submit your next manuscript at $>$ springeropen.com 